\title{
Joint Bandwidth and Power Allocation for MIMO Two-Way Relays-Assisted Overlay Cognitive Radio Systems
}

\author{
Ahmad Alsharoa, Student Member, IEEE, Hakim Ghazzai, Member, IEEE, Elias Yaacoub, Senior Member, \\ IEEE, Mohamed-Slim Alouini, Fellow, IEEE, and Ahmed E. Kamal, Fellow, IEEE
}

\begin{abstract}
This paper studies the achievable cognitive sum rate of an overlay cognitive radio (CR) system assisted with multiple antennas two-way relays in which primary users (PUs) cooperate with cognitive users (CUs) for mutual benefits. In this context, the problem of both bandwidth and power allocation is investigated. We propose that the CUs are allowed to allocate a part of the PUs spectrum to perform their cognitive transmission. In return, acting as amplify-and-forward two-way relays, they are exploited to support PUs to reach their target data rates over the remaining bandwidth. Power expressions for optimal transmit power allocated per $P U$ and $C U$ antenna are derived under primary quality-of-service constraint in addition to bandwidth and power budget constraints. More specifically, CUs act as relays for the PUs transmission and gain some spectrum as long as they respect these constraints. After deriving the optimal transmit powers, we employ a strong optimization tool based on swarm intelligence to optimize the full and complex relay amplification gain matrices in addition to the bandwidths released to primary and cognitive transmission. Furthermore, three different utility functions are considered in our optimization problems depending on the level of fairness among CUs.
\end{abstract}

Index Terms-Overlay cognitive radio systems, two-way relaying technique, multiple-input multiple-output antennas.

\section{INTRODUCTION}

\section{A. Background}

Cognitive radio (CR) is proposed as an intelligent novel approach to solve the spectrum deficit problem [1]. The basic idea of $\mathrm{CR}$ is that cognitive users (CUs) are allowed to utilize the spectrum of licensed users which are also known as primary users (PUs) in an opportunistic fashion. CR can be grouped into three main techniques; interweave, underlay, and overlay [2]. The original idea of cognitive radio was based on the interweave technique, where the CUs are allowed to access the primary spectrum only when the PUs are inactive

A part of this work has been presented at IEEE Global Communications Conference (IEEE Globecom 2014), Austin, Texas, USA.

This work was made possible by grant NPRP 5-250-2-087 from the Qatar National Research Fund (a member of The Qatar Foundation). The statements made herein are solely the responsibility of the authors.

Ahmad Alsharoa, and Ahmed E. Kamal are with Iowa State University (ISU), Ames, Iowa, USA, E-mail: \{alsharoa, kamal\}@iastate.edu.

Hakim Ghazzai is with Qatar Mobility Innovations Center (QMIC), Qatar Science and Technology Park, Doha, Qatar. E-mail: hakimg@qmic.com.

Elias Yaacoub is with the Strategic Decision Group (SDG), and with the Lebanon Faculty of Computer Studies, Arab Open University (AOU), Beirut, Lebanon. (E-mail: eliasy@ieee.org).

Mohamed-Slim Alouini is with the Computer, Electrical and Mathematical Sciences and Engineering (CEMSE) Division, King Abdullah University of Science and Technology (KAUST), Thuwal, Makkah Province, Saudi Arabia. E-mails: slim.alouini@kaust.edu.sa.
[3]. In the underlay technique, CUs access the spectrum simultaneously with PUs under some interference limitation constraints to maintain a certain primary quality-of-service (QoS) [4]. In the overlay technique, CUs are allowed to use a part of their resources to enhance the primary signal. In counterpart, the PUs may release some bandwidths for CUs data transmission [5]. This technique can be exploited with cooperative relaying techniques to enhance the system performance.

Two-way relaying (TWR), has lately attracted a lot of attention in the literature. In conventional TWR, exchanging different messages between two terminals takes place into two phases only instead of four phases in the traditional one-way relaying. In the first phase, the users transmit their signals simultaneously to the relays. Subsequently, in the second phase, the relays broadcast the signal to the users [6]. The authors in [7] investigated the performance of the TWR transmission and compared them to the traditional one-way relaying transmission. To perform this, several relay strategies are used, in our framework, we are interested in amplify-andforward (AF) protocol, where the relay amplifies the received signal before broadcasting it to the destination, due to its low computational complexity and low delay in the relay node (i.e., AF allows faster transmission without processing delay). Moreover, AF requires much less computing power compared to other protocols.

The multiple-input multiple-output (MIMO) technique provides more degrees of freedom to the system to enhance the system throughput. Various studies have employed MIMO antennas with TWR [8], [9]. Moreover, many studies employed TWR system in conjunction with interweave and underlay cognitive modes in order to minimize the total power consumption or to enhance the cognitive sum rate while respecting the primary QoS, respectively, [10], [11]. The work in [10] and [11] compared the single and multiple antenna cases in underlay cognitive scenario.

\section{B. Overlay Cognitive Radio: Literature Review}

Leasing model, which is also called property rights model, can be categorized into two categories; spectrum leasing and time leasing. For spectrum leasing, PUs who own the spectrum can possibly lease a part of the spectrum to CUs for appropriate remuneration and can exploit the existence of CUs to enhance their performance and QoS. In counterpart, CUs can use the leased spectrum for their own transmission 
by performing decentralized power control [12], [13]. On the other hand, for time leasing, PUs can lease a portion of their time for CUs' transmission. In return, in the remaining time, CUs help the PUs to enhance their performance. Indeed, the data transmission takes place in three time slots, in the first time slot, PUs transmit their signals to CUs, while in the second time slot, CUs broadcast the primary signal to the primary destination, finally, the CUs use the remaining time slot for their own transmission [14], [15]. In this paper, the "overlay" model term is used to indicate the spectrum leasing model.

Overlay $\mathrm{CR}$ technique has been introduced in literature as a solution to enhance the spectral efficiency of primary transmissions while exploiting the existence of CUs [16]. Most of the studies model CUs as one-way relays that decodeand-forward the primary signals to the destination in order to improve the system reliability. This operation requires the knowledge of the primary user's data sequence and/or codebook [16]. Furthermore, in overlay CR, CUs need to know the primary channel gains in addition to encoding techniques if they will decode the primary signal. However, compared to interweave and underlay CR, overlay offers the possibility to freely transmit their signals without any constraints in terms of time and transmitted power. It just requires that PUs know the existence of overlaying cognitive relays in order to coordinate their transmissions. Note here that priority is given to PUs in contrast with the non-cognition case where relays have the unique role of forwarding the primary signals.

Few work have employed TWR systems with overlay CR technique [17]-[21]. These studies assume the absence of direct link between primary terminals and utilize CU relays to forward their signals simultaneously. This scenario overcomes the hidden terminal problem which happens when the PUs are shadowed or are in severe multipath fading. In return, CUs are allowed to share a part of the primary bandwidth to perform their communication. This spectrum sharing scenario might involves some forms of coordination and cooperation between the two types of users (i.e., primary and secondary users), mainly when this cooperation is optimized. For instance, this can be implemented when all users belong to the same network as suggested in [17]. The authors have considered a device-to-device (D2D) communication scenario overlaying a cellular network where D2D users, playing the role of CUs, communicate bi-directionally with each other while assisting the two-way communications between a cellular user and its base station. The work in [18] and [19] proposed a typical model comprising a pair of PUs and a pair of CUs. The objective was to find an optimal power allocation at the single relaying cognitive node that minimizes the outage probability at the cognitive receiver for given outage constraints on the primary system. A joint relay selection and resource allocation algorithm for TWR overlay CR networks is also proposed in [20], where the best relays (CUs) with higher channel gain are selected to act as relays to help for primary transmission.

\section{Contributions}

In this paper, we investigate the problem of bandwidth and power allocation for TWR-MIMO overlay cognitive networks using multiple AF relays. The contributions of this paper can be summarized as follows:

- We formulate an optimization problem that maximizes the TWR-MIMO overlay cognitive rate while taking into account all transceiver power budgets in addition to the PU QoS requirements. In our framework, we assume that each $\mathrm{CU}$ is allowed to share the primary bandwidth in order to perform its transmission. In return, they are engaged to complete the primary transmission by amplifying and forwarding the PU data over the remaining bandwidth.

- Due to the non-convexity of the problem, we firstly derive expressions of the transmit powers allocated to primary and cognitive users for a fixed user bandwidth and relay amplification gain. Then, we employ a meta-heuristic approach based on particle swarm optimization (PSO) algorithm to find sub-optimal CU bandwidth allocation in addition to the relay amplification matrix gains.

- We consider different cognitive objective functions depending on the level of fairness among CUs.

- Finally, we analyze the performance of our scheme under different system parameters and we compare the algorithm in terms of convergence speed and computational complexity with a recently proposed heuristic approach entitled the grey wolf optimizer (GWO) [22].

\section{Paper Organization}

The remainder of this paper is organized as follows. Section II presents the MIMO-TWR overlay cognitive system model using AF relays. The problem formulation is described in Section III. Section IV gives optimal power expressions for the different considered utilities and describes the PSO algorithm for bandwidth and relay gain optimization. The numerical results are discussed in Section V. Finally, the paper is concluded in Section VI.

We need to make some notes about the notations used in this paper. The superscripts $(.)^{T}$ and $(.)^{H}$ correspond to the transpose and the hermitian operator, respectively. $\mathbb{C}$ and $\mathbb{R}$ denote the field of complex and real numbers, respectively. $\mathbb{E}($.$) and \operatorname{Tr}($.$) denote the expectation and the trace operator,$ respectively. $(x)^{+}$denotes a maximum between $x$ and zero.

\section{SySTEM MOdEL}

We consider an overlay half duplex CR network with two primary users $\mathrm{PU}_{1}$ and $\mathrm{PU}_{2}$ in addition to a cognitive network consisting of $L \mathrm{CUs}$ and one cognitive base station. All nodes are equipped with $M$ antennas. A non-line of sight link between $\mathrm{PU}_{1}$ and $\mathrm{PU}_{2}$ is considered as illustrated in Fig. 1. The $L$ CUs act as two-way relays for the PUs over the primary bandwidth. In exchange, the PUs may release some of their bandwidths to the CUs to accomplish their own data transmission as long as the PUs maintain their QoS.

Let $T$ denote the time duration that a primary user is allowed to transmit data over the bandwidth $B_{\text {tot }}$. In our overlay MIMO-CR scheme, we assume that the total bandwidth is divided into $L+1$ fractions denoted $B_{0}, B_{1}, \ldots, B_{L}$, where the primary transmission is held over $B_{0}$ while for each $l^{\text {th }} \mathrm{CU}$, 
we allocate the bandwidth fraction $B_{l}$ such that there is no inter-user interference between all the primary and secondary nodes $\left(\sum_{l=0}^{L} B_{l}=B_{\mathrm{tot}}\right)$, as shown in Fig. 2 .

Assuming independent and identically distributed (i.i.d) complex Gaussian signals, PUs exchange their messages via $L$ CUs as follows: In the first phase, both $\mathrm{PU}_{1}$ and $\mathrm{PU}_{2}$ transmit their messages $\boldsymbol{x}_{1}$ and $\boldsymbol{x}_{2}$ simultaneously to the $L$ CUs with a covariance power matrix of vector $\boldsymbol{x}_{t}$ denoted $\boldsymbol{P}_{t}=\mathbb{E}\left(\boldsymbol{x}_{t} \boldsymbol{x}_{t}^{H}\right)$ Watt/Hz (power per unit frequency), where $t=\{1,2\}$. Perfect synchronization between $\mathrm{PU}_{1}$ and $\mathrm{PU}_{2}$ is assumed [23]. In the second phase, the CUs play the role of relays by transmitting the amplified signal to the PUs with a covariance power matrix denoted $\boldsymbol{P}_{r_{l}}$ Watt/Hz, where $l=1, \ldots, L$. During PUs transmission and reception, the CUs transmit their data $\boldsymbol{x}_{c_{l}}$ to the cognitive base station over the remaining bandwidth (i.e., $B_{1}, \ldots, B_{L}$ ) with a covariance power matrix denoted $\boldsymbol{P}_{c_{l}}=\mathbb{E}\left(\boldsymbol{x}_{c_{l}} \boldsymbol{x}_{c_{l}}^{H}\right)$ Watt/Hz, where $l=1, \ldots, L$.

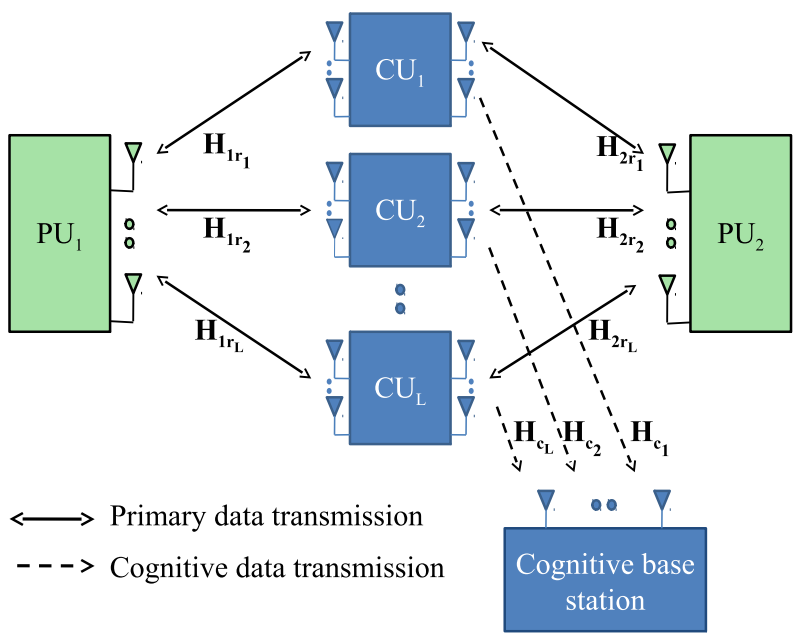

Fig. 1 Overlay TWR-MIMO system model.

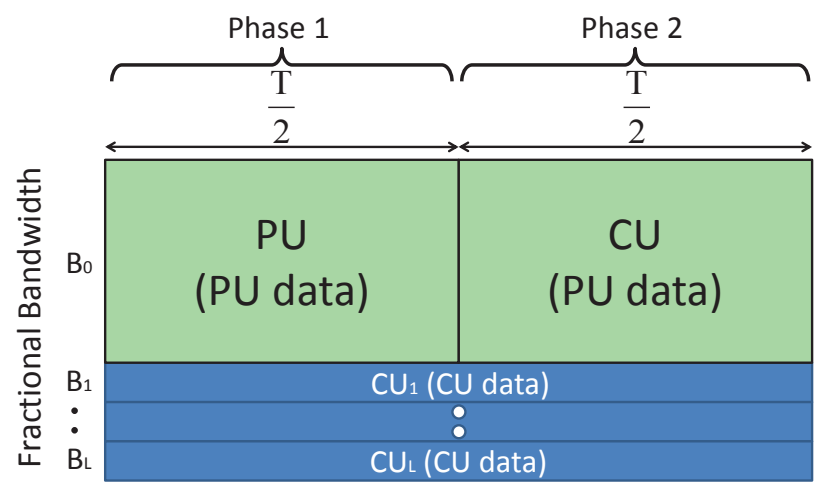

Fig. 2 Time-bandwidth allocation.

Let us define $\bar{E}_{p}$ and $\bar{E}_{c}$ as the peak energy at each PU and the peak energy at each CU, respectively. $\boldsymbol{H}_{1 r_{l}} \in$ $\mathbb{C}^{M \times M}, \boldsymbol{H}_{2 r_{l}} \in \mathbb{C}^{M \times M}$, and $\boldsymbol{H}_{c_{l}} \in \mathbb{C}^{M \times M}$ are the MIMO channel gains in the first time slot between $\mathrm{PU}_{1}$ and the $l^{\text {th }}$ $\mathrm{CU}, \mathrm{PU}_{2}$ and the $l^{\text {th }} \mathrm{CU}$, and the $l^{\text {th }} \mathrm{CU}$ and the cognitive base station, respectively. Where in the second time slot the MIMO channel gains are denoted by $\hat{\boldsymbol{H}}_{1 r_{l}} \in \mathbb{C}^{M \times M}, \hat{\boldsymbol{H}}_{2 r_{l}} \in$ $\mathbb{C}^{M \times M}$, and $\hat{\boldsymbol{H}}_{c_{l}} \in \mathbb{C}^{M \times M}$. All the channel gains are assumed to be i.i.d fading channel gains and constant during the coherence time. If the channels are highly correlated during two consecutive time slots, then channel reciprocity is assumed i.e., $\boldsymbol{H}_{1 r_{l}}=\hat{\boldsymbol{H}}_{1 r_{l}}, \boldsymbol{H}_{2 r_{l}}=\hat{\boldsymbol{H}}_{2 r_{l}}, \boldsymbol{H}_{c_{l}}=\hat{\boldsymbol{H}}_{c_{l}}$ In addition to that, perfect channel state information at transmitters and receivers are considered. Without loss of generality, all the noise variances are assumed to be equal to $\mathcal{N}_{0}$.

Let $\boldsymbol{V}_{t}$ and $\boldsymbol{U}_{t}$, where $t \in\{1,2\}$ be two unitary precoder and decoder matrices, respectively, employed by PUs. In the first phase, $\mathrm{PU}_{t}$ employs the precoder matrix $\boldsymbol{V}_{t}$ such as: $\boldsymbol{x}_{t}=$ $\boldsymbol{V}_{t} \tilde{\boldsymbol{x}}_{t}$ where $\boldsymbol{x}_{t}$ is the transmitted signal after being precoded by $\mathrm{PU}_{t}$. Subsequently, during the second phase, $\mathrm{PU}_{t}$ employs the decoder matrix $\boldsymbol{U}_{t}$ such as: $\boldsymbol{r}_{t}=\boldsymbol{U}_{t}^{H} \boldsymbol{y}_{t}$, where $\boldsymbol{y}_{t}$ and $\boldsymbol{r}_{t}$ are the received signals at $\mathrm{PU}_{t}$ before and after decoding, respectively. The choice of $\boldsymbol{V}_{t}$ and $\boldsymbol{U}_{t}$ will be defined later.

The approach of the paper was initially designed to a scenario where PUs and CUs belong to the same legacy system; e.g. LTE with LTE device-to-device (D2D) communications. In the case where it must be extended to deal with different legacy scenario, then the following rules apply:

- Each CU needs to support both standards.

- Each CU needs to have two separate RF chains: one to be used for the secondary transmissions over the secondary system (e.g., 802.11ac standard) and one to be used over the primary system (e.g., LTE standard) to relay PUs' data.

- This requires doubling the number of antennas since transmission over both systems will be simultaneous: If $M$ is the current number of antennas in the paper, each CU would need " $2 M$ " antennas: $M$ to be used over the primary system (LTE) and another $M$ to be used over the secondary system.

- This will lead to increase the costs of the CU devices, but they would still be able to use the spectrum for free.

- It should be noted that the different legacy scenario assumes that the two standards use overlapping spectrum bands. Otherwise, PUs cannot share a portion of their bandwidth with CUs, since the technology used by CUs would be operational on different frequency bands.

\section{PROBLEM Formulation}

In this section, we formulate an optimization problem that maximizes the cognitive objective function for multiple MIMO TWR-CR networks while satisfying the required QoS of the PUs. Different utility metrics leading to different bandwidth and power allocation are presented and discussed depending on the cognitive objective. Without loss of generality, channel reciprocity is assumed.

\section{A. Primary Data Rate}

In the first phase, the baseband received signal at the $l^{\text {th }}$ $\mathrm{CU}$ over $B_{0}$ is given as follows

$$
\boldsymbol{y}_{r_{l}}=\boldsymbol{H}_{1 r_{l}} \boldsymbol{x}_{1}+\boldsymbol{H}_{2 r_{l}} \boldsymbol{x}_{2}+\boldsymbol{n}_{r_{l}}
$$


where $\boldsymbol{n}_{\boldsymbol{r}_{\boldsymbol{i}}}$ is the additive Gaussian noise at the $l^{\text {th }}$ relay and $\boldsymbol{x}_{t}$ is the transmitted signal after precoding by $\mathrm{PU}_{t}$, where $t \in\{1,2\}$. During the second phase, each relay CU amplifies $\boldsymbol{y}_{r_{l}}$ by multiplying it by a full matrix $\boldsymbol{G}_{l} \in \mathbb{C}^{M \times M}$ and broadcasting it to the PUs. Finally, the received signals at $\mathrm{PU}_{1}$ and $\mathrm{PU}_{2}$ are respectively given as

$$
\begin{aligned}
& \boldsymbol{y}_{1}=\underbrace{\hat{\boldsymbol{\Phi}} \boldsymbol{x}_{1}}_{\text {Self Interference }}+\boldsymbol{\Phi} \boldsymbol{x}_{2}+\boldsymbol{z}_{1}, \\
& \boldsymbol{y}_{2}=\boldsymbol{\Psi} \boldsymbol{x}_{1}+\underbrace{\hat{\boldsymbol{\Psi}} \boldsymbol{x}_{2}}_{\text {Self Interference }}+\boldsymbol{z}_{2},
\end{aligned}
$$

where $\boldsymbol{\Phi}=\sum_{l=1}^{L} \boldsymbol{H}_{1 r_{l}}^{T} \boldsymbol{G}_{l} \boldsymbol{H}_{2 r_{l}}, \hat{\boldsymbol{\Phi}}=\sum_{l=1}^{L} \boldsymbol{H}_{1 r_{l}}^{T} \boldsymbol{G}_{l} \boldsymbol{H}_{1 r_{l}}, \boldsymbol{\Psi}=$ $\sum_{l=1}^{L} \boldsymbol{H}_{2 r_{l}}^{T} \boldsymbol{G}_{l} \boldsymbol{H}_{1 r_{l}}$, and $\hat{\boldsymbol{\Psi}}=\sum_{l=1}^{L} \boldsymbol{H}_{2 r_{l}}^{T} \boldsymbol{G}_{l} \boldsymbol{H}_{2 r_{l}}$, are the equivalent MIMO channels obtained at $\mathrm{PU}_{1}$ and $\mathrm{PU}_{2}$, respectively before decoding. $\boldsymbol{z}_{t}=\sum_{l=1}^{L}\left(\boldsymbol{H}_{t r_{l}}^{T} \boldsymbol{G}_{l} \boldsymbol{n}_{r_{l}}\right)+\boldsymbol{n}_{t}$ and $\boldsymbol{n}_{t}$ are the equivalent amplified noise at $\mathrm{PU}_{\mathrm{t}}$ before decoding and the additive Gaussian noise vectors at $\mathrm{PU}_{\mathrm{t}}$, respectively, where $t \in\{1,2\}$. Using the knowledge of the channel information and channel reciprocity, the PUs can remove the self interference by eliminating their own signals (i.e., $\boldsymbol{x}_{1}$ for $\mathrm{PU}_{1}$ and $\boldsymbol{x}_{2}$ for $\mathrm{PU}_{2}$ ). Thus, the received signal $\boldsymbol{r}_{1}$ and $\boldsymbol{r}_{2}$ after employing the decoders $\boldsymbol{U}_{t}$ are given, respectively, by

$$
\begin{aligned}
& \boldsymbol{r}_{1}=\boldsymbol{U}_{1}^{H} \boldsymbol{\Phi} \boldsymbol{x}_{2}+\tilde{\boldsymbol{z}}_{1}=\boldsymbol{U}_{1}^{H} \boldsymbol{\Phi} \boldsymbol{V}_{2} \tilde{\boldsymbol{x}}_{2}+\tilde{\boldsymbol{z}}_{1} \\
& \boldsymbol{r}_{2}=\boldsymbol{U}_{2}^{H} \boldsymbol{\Psi} \boldsymbol{x}_{1}+\tilde{\boldsymbol{z}}_{2}=\boldsymbol{U}_{2}^{H} \boldsymbol{\Psi} \boldsymbol{V}_{1} \tilde{\boldsymbol{x}}_{1}+\tilde{\boldsymbol{z}}_{2}
\end{aligned}
$$

where $\tilde{\boldsymbol{z}}_{t}=\boldsymbol{U}_{t}^{H} \boldsymbol{z}_{t}$ is the equivalent amplified noise at $\mathrm{PU}_{\mathrm{t}}$ after decoding. The covariance matrix of the noise $\tilde{\boldsymbol{z}}_{t}$ can be given as

$$
\begin{aligned}
\boldsymbol{C}_{\tilde{\boldsymbol{z}}_{t}} & =\mathbb{E}\left[\tilde{\boldsymbol{z}}_{t} \tilde{\boldsymbol{z}}_{t}^{H}\right] \\
& =\mathcal{N}_{0} \sum_{l=1}^{L} \boldsymbol{U}_{t}^{H} \boldsymbol{H}_{t r_{l}}^{T} \boldsymbol{G}_{l}\left(\boldsymbol{U}_{t}^{H} \boldsymbol{H}_{t r_{l}}^{T} \boldsymbol{G}_{l}\right)^{H}+\mathcal{N}_{0} \mathbf{I}_{M},
\end{aligned}
$$

where $\mathrm{I}_{M}$ denotes the identity matrix of size $M$.

Let us now define the unitary precoding and decoding matrices using the singular value decomposition (SVD) which converts the MIMO channel into parallel channels characterized by their associated eigenmodes. Thus, we perform SVDs for the matrices $\boldsymbol{\Phi}$ and $\boldsymbol{\Psi}$ as follows: $\boldsymbol{\Phi}=\boldsymbol{U}_{1} \boldsymbol{S}_{\Phi} \boldsymbol{V}_{2}^{H}$ and $\boldsymbol{\Psi}=\boldsymbol{U}_{2} \boldsymbol{S}_{\Psi} \boldsymbol{V}_{1}^{H}$, where $\boldsymbol{S}_{\Phi}$ and $\boldsymbol{S}_{\Psi}$ are diagonal matrices with square roots of the eigenvalues of matrix $\boldsymbol{\Phi}$ and $\boldsymbol{\Psi}$, respectively. As such, the primary rates of the $\mathrm{PU}_{1}$ and $\mathrm{PU}_{2}$ after SVD can be respectively given as

$$
\begin{aligned}
& R_{p_{1}}=\frac{B_{0}}{2} \sum_{m=1}^{M} \log _{2}\left(1+\frac{\boldsymbol{S}_{\Phi}^{2}(m, m) \boldsymbol{P}_{2}(m, m)}{\boldsymbol{C}_{\tilde{\boldsymbol{z}}_{1}}(m, m)}\right), \\
& R_{p_{2}}=\frac{B_{0}}{2} \sum_{m=1}^{M} \log _{2}\left(1+\frac{\boldsymbol{S}_{\Psi}^{2}(m, m) \boldsymbol{P}_{1}(m, m)}{\boldsymbol{C}_{\tilde{\boldsymbol{z}}_{2}}(m, m)}\right) .
\end{aligned}
$$

The factor $\frac{1}{2}$ is added as primary transmission is held over two time slots. In order to meet the target transmission rate for the primary network, $R_{p_{1}}[\mathrm{bits} / \mathrm{s}]$ and $R_{p_{2}}$ [bits/s] should be no less than the primary target transmission rate $R_{0}[\mathrm{bits} / \mathrm{s}$, i.e.,

$$
R_{p_{1}} \geq \alpha R_{0} \quad \text { and } \quad R_{p_{2}} \geq(1-\alpha) R_{0}
$$

where $\alpha \in[0,1]$ is the rate profile of the system to characterize the boundary rates at the PUs. From (9), we can show that the fractional bandwidth needed for the primary network should satisfy

$$
\begin{array}{r}
B_{0} \geq \max \left(\frac{2 \alpha R_{0}}{\sum_{m=1}^{M} \log _{2}\left(1+\frac{\boldsymbol{S}_{\Phi}^{2}(m, m) \boldsymbol{P}_{2}(m, m)}{\boldsymbol{C}_{\tilde{\boldsymbol{z}}_{1}}(m, m)}\right)},\right. \\
\left.\frac{2(1-\alpha) \boldsymbol{R}_{0}}{\sum_{m=1}^{M} \log _{2}\left(1+\frac{\boldsymbol{S}_{\Psi}^{2}(m, m) \boldsymbol{P}_{1}(m, m)}{\boldsymbol{C}_{\tilde{z}_{2}}(m, m)}\right)}\right) .
\end{array}
$$

Therefore, the PUs may release the remaining fractional bandwidth $\left(B_{\text {tot }}-B_{0}\right)$ to CUs.

\section{B. Secondary Data Rate}

On other hand, the received signal at the cognitive base station from the $l^{\text {th }} \mathrm{CU}$ over bandwidth $B_{l}$ can be given as

$$
\boldsymbol{y}_{c_{l}}=\boldsymbol{H}_{c_{l}} \boldsymbol{x}_{c_{l}}+\boldsymbol{n}_{c_{l}},
$$

where $\boldsymbol{n}_{c_{l}}$ is the additive Gaussian noise at the cognitive base station. Thus, the received signal $\boldsymbol{r}_{c_{l}}$ after decoding is given by

$$
\boldsymbol{r}_{c_{l}}=\boldsymbol{U}_{c_{l}}^{H} \boldsymbol{H}_{c_{l}} \boldsymbol{x}_{c_{l}}+\boldsymbol{U}_{c_{l}}^{H} \boldsymbol{n}_{c_{l}}=\boldsymbol{U}_{c_{l}}^{H} \boldsymbol{H}_{c_{l}} \boldsymbol{V}_{c_{l}} \tilde{\boldsymbol{x}}_{c_{l}}+\boldsymbol{U}_{c_{l}}^{H} \boldsymbol{n}_{c_{l}} .
$$

Define $\boldsymbol{H}_{c_{l}}=\boldsymbol{U}_{c_{l}} \boldsymbol{S}_{c_{l}} \boldsymbol{V}_{c_{l}}^{H}$, where $\boldsymbol{S}_{c_{l}}$ is a diagonal matrix with square roots of the eigenvalues of matrix $\boldsymbol{H}_{c_{l}}$. Therefore, the cognitive rate of $l^{\text {th }} \mathrm{CU}$ at the cognitive base station can be expressed in [bits/s] as

$$
R_{c_{l}}=B_{l} \sum_{m=1}^{M} \log _{2}\left(1+\frac{\boldsymbol{S}_{c_{l}}^{2}(m, m) \boldsymbol{P}_{c_{l}}(m, m)}{N_{0}}\right) .
$$

\section{Optimization Problem}

Recall that the PU and CU users have as energy budgets $\bar{E}_{p}$ and $\bar{E}_{c}$ expressed in Joules, respectively, and that the power budgets of PU and CU equal to $\bar{P}_{p}$ and $\bar{P}_{c}$ Watt, respectively. Thus, the energy budget constraints at the $t^{t h} \mathrm{PU}$ and $l^{\text {th }} \mathrm{CU}$ are over time duration $T$ given respectively as

$$
\begin{aligned}
& \frac{T}{2} B_{0} \operatorname{Tr}\left(\boldsymbol{P}_{t}\right) \leq \bar{E}_{p}, \\
& T B_{l} \operatorname{Tr}\left(\boldsymbol{P}_{c_{l}}\right)+\frac{T}{2} B_{0} \operatorname{Tr}\left(\boldsymbol{\Omega}_{1 r_{l}} \boldsymbol{P}_{1} \boldsymbol{\Omega}_{1 r_{l}}^{H}+\boldsymbol{\Omega}_{2 r_{l}} \boldsymbol{P}_{2} \boldsymbol{\Omega}_{2 r_{l}}^{H}\right. \\
&\left.+N_{0} \boldsymbol{G}_{l} \boldsymbol{G}_{l}^{H}\right) \leq \bar{E}_{c} .
\end{aligned}
$$

or equivalently

$$
\begin{aligned}
& \frac{B_{0}}{2} \operatorname{Tr}\left(\boldsymbol{P}_{t}\right) \leq \bar{P}_{p}, \\
& B_{l} \operatorname{Tr}\left(\boldsymbol{P}_{c_{l}}\right)+\frac{B_{0}}{2} \operatorname{Tr}\left(\boldsymbol{\Omega}_{1 r_{l}} \boldsymbol{P}_{1} \boldsymbol{\Omega}_{1 r_{l}}^{H}+\boldsymbol{\Omega}_{2 r_{l}} \boldsymbol{P}_{2} \boldsymbol{\Omega}_{2 r_{l}}^{H}\right. \\
&\left.\quad+N_{0} \boldsymbol{G}_{l} \boldsymbol{G}_{l}^{H}\right) \leq \bar{P}_{c} .
\end{aligned}
$$


where $\boldsymbol{\Omega}_{1 r_{l}}=\boldsymbol{G}_{l} \boldsymbol{H}_{1 r_{l}}$ and $\boldsymbol{\Omega}_{2 r_{l}}=\boldsymbol{G}_{l} \boldsymbol{H}_{2 r_{l}}$ are the MIMO equivalent channel gains.

Let $U\left(R_{c_{l}}\right)$ denote the rate utility of the cognitive system. Thus, the optimization problem of MIMO TWR-CR with multiple relays that maximizes the rate utility while satisfying specific power budgets and target primary rate constraints can be formulated as

$$
\underset{\boldsymbol{B}, \boldsymbol{P}_{1}, \boldsymbol{P}_{2}, \boldsymbol{P}_{c_{l}}, \boldsymbol{G}_{l} \geq 0}{\operatorname{maximize}} U\left(R_{c_{l}}\right)
$$

subject to:

(C1: Power budget constraints at the primary users):

$$
0 \leq \frac{B_{0}}{2} \operatorname{Tr}\left(\boldsymbol{P}_{t}\right) \leq \bar{P}_{p}, \quad \forall t=1,2,
$$

(C2: Power budget constraints at the cognitive users):

$$
\begin{aligned}
B_{l} \operatorname{Tr}\left(\boldsymbol{P}_{c_{l}}\right)+ & \frac{B_{0}}{2} \operatorname{Tr}\left(\boldsymbol{\Omega}_{1 r_{l}} \boldsymbol{P}_{1} \boldsymbol{\Omega}_{1 r_{l}}^{H}+\boldsymbol{\Omega}_{2 r_{l}} \boldsymbol{P}_{2} \boldsymbol{\Omega}_{2 r_{l}}^{H}\right. \\
& \left.+N_{0} \boldsymbol{G}_{l} \boldsymbol{G}_{l}^{H}\right) \leq \bar{P}_{c}, \quad \forall l=1, \ldots, L,
\end{aligned}
$$

(C3: Rate constraint for the first primary user):

$$
R_{p_{1}} \geq \alpha R_{0},
$$

(C4: Rate constraint for the second primary user):

$$
R_{p_{2}} \geq(1-\alpha) R_{0},
$$

(C5: Total bandwidth constraint):

$$
\sum_{l=0}^{L} B_{l}=B_{\mathrm{tot}} \text {, }
$$

where $\boldsymbol{B}=\left[B_{0}, B_{1}, \ldots, B_{L}\right]$ is the vector that contains the fractions of bandwidth assigned to primary and cognitive transmissions. Constraints (17) and (18) represent the peak energy constraints at PUs and CUs, respectively. The term $\operatorname{Tr}\left(\boldsymbol{\Omega}_{1 r_{l}} \boldsymbol{P}_{1} \boldsymbol{\Omega}_{1 r_{l}}^{H}+\boldsymbol{\Omega}_{2 r_{l}} \boldsymbol{P}_{2} \boldsymbol{\Omega}_{2 r_{l}}^{H}+N_{0} \boldsymbol{G}_{l} \boldsymbol{G}_{l}^{H}\right)$ in constraint (18) is equivalent to the relay amplified power of the $l^{\text {th }} \mathrm{CU}$.

\section{Utility Selection}

In this section, we characterize three different utility metrics that will be employed in the optimization problem (16).

Max C/I Utility:

The utility of this metric is equivalent to the sum data rate of the cognitive network $U\left(R_{c_{l}}\right)=\sum_{l=1}^{L} R_{c_{l}}$. This approach is known in the literature as Max C/I [24] as it promotes users with favorable channel and interference conditions by allocating to them most of the resources, whereas users suffering from higher propagation losses and/or interference levels will be deprived from the bandwidth as well as the power and will have very low data rates. Note that, thanks to the employed overlay scheme, and thus, the elimination of user interference, the Max C/I utility promotes users with favorable channels conditions only.

\section{Max-Min Utility:}

Due to the unfairness of Max $\mathrm{C} / \mathrm{I}$ resource allocation, the need for more fair utility metrics arises. Max-Min utilities are a family of utility functions attempting to maximize the minimum data rate in the network $U\left(R_{c_{l}}\right)=\min _{l}\left(R_{c_{l}}\right)$ [25]. By increasing the priority of users having lower rates, Max-Min utilities lead to more fairness in the network. In order to simplify the problem for this approach, we define a new decision variable $R_{\min }=\min _{l}\left(R_{c_{l}}\right)$. Therefore, our optimization problem becomes

$$
\underset{\boldsymbol{B}, \boldsymbol{P}_{1}, \boldsymbol{P}_{2}, \boldsymbol{P}_{c}, \boldsymbol{G}_{l}, R_{\min } \geq 0}{\operatorname{maximize}} R_{\min }
$$

subject to:

$$
\begin{gathered}
R_{c_{l}} \geq R_{\min } \quad \forall l=1, \ldots, L, \\
(\mathrm{C} 1),(\mathrm{C} 2),(\mathrm{C} 3),(\mathrm{C} 4),(\mathrm{C} 5) .
\end{gathered}
$$

\section{Proportional Fair Utility:}

A tradeoff between the maximization of the sum rate and the maximization of the minimum rate could be the maximization of the geometric mean data rate $U\left(R_{c_{l}}\right)=\left(\prod_{l=1}^{L} R_{c_{l}}\right)^{1 / L}$ [26]. The proportional fair (PF) metric is fair, since a user with a data rate close to zero will make the whole product go to zero. Hence, any algorithm maximizing the geometric means would avoid having any user with very low data rate. In addition to this, the metric will reasonably promote users with good wireless channels (capable of achieving high data rates), since a high data rate will contribute in increasing the product.

\section{Optimal Power Allocation and Particle SWARM OPTIMIZATION ALGORITHM}

The formulated optimization problem is a non-convex problem and its optimal solution remains unsolved. For this reason, we propose to solve it in two steps. In the first step, we derive power expressions at each iteration for the optimal transmit primary powers (i.e., $\boldsymbol{P}_{1}$ and $\boldsymbol{P}_{2}$ ) and cognitive transmit powers (i.e., $\boldsymbol{P}_{\boldsymbol{c}_{l}}$ ) by assuming fixed bandwidths of both primary and cognitive users and fixed amplification matrix gains (equivalent to fixed relay power), at all CUs. As a result, we convert our formulated problem to a convex one. The primal-dual method is used due to its simplicity and to the fact that it provides an expression of the power allocation per each antenna for the different utility functions. This can help in interpreting the behavior of each terminal in the network thanks to the water-filling expressions that will be derived next. Then, we propose to employ the subgradient method in order to optimize the Lagrangian multipliers. Note that the interior-point method could be also employed to solve the problem by finding numerically the optimal solution using the Newton method. Although the convergence of the subgradient method is slower than the interior-point method, the subgradient method remains competitive mainly for large scale problems as it requires little storage [27]. In the second step, we employ swarm intelligence to jointly optimize the system bandwidths with the $\mathrm{CU}$ amplification gain matrices.

\section{A. Optimal Transmit Power Allocation}

We can solve our convex optimization problem for fixed $\boldsymbol{B}$ and $\boldsymbol{G}_{l}, \forall l=1, \ldots, L$, by exploiting its strong duality as follows [27]:

$$
\underset{\boldsymbol{\lambda} \geq 0}{\operatorname{minimum}} \underset{\boldsymbol{P}_{1}, \boldsymbol{P}_{2}, \boldsymbol{P}_{c_{l}} \geq 0}{\operatorname{maximum}} \mathcal{L}\left(\boldsymbol{\lambda}, \boldsymbol{P}_{1}, \boldsymbol{P}_{2}, \boldsymbol{P}_{c_{l}}\right),
$$




$$
\begin{aligned}
& \mathcal{L}\left(\boldsymbol{\lambda}, \boldsymbol{P}_{1}, \boldsymbol{P}_{2}, \boldsymbol{P}_{c}\right)=U\left(R_{c_{l}}\right)-\sum_{t=1}^{2} \lambda_{p_{t}}\left(\frac{B_{0}}{2} \operatorname{Tr}\left(\boldsymbol{P}_{t}\right)-\bar{P}_{p}\right)-\sum_{l=1}^{L} \lambda_{c_{l}}\left(B_{l} \operatorname{Tr}\left(\boldsymbol{P}_{c_{l}}\right)+\frac{B_{0}}{2} \operatorname{Tr}\left(\boldsymbol{\Omega}_{1 r_{l}} \boldsymbol{P}_{1} \boldsymbol{\Omega}_{1 r_{l}}^{H}+\boldsymbol{\Omega}_{2 r_{l}} \boldsymbol{P}_{2} \boldsymbol{\Omega}_{2 r_{l}}^{H}\right.\right. \\
& \left.\left.+N_{0} \boldsymbol{G}_{l} \boldsymbol{G}_{l}^{H}\right)-\bar{P}_{c}\right)+\lambda_{t h_{1}}\left(R_{p_{1}}-\alpha R_{0}\right)+\lambda_{t h_{2}}\left(R_{p_{2}}-(1-\alpha) R_{0}\right)-\lambda_{B}\left(\sum_{l=0}^{L} B_{l}-B_{\mathrm{tot}}\right)+\sum_{l=1}^{L} \lambda_{R_{l}}\left(R_{c_{l}}-R_{\mathrm{min}}\right)
\end{aligned}
$$

$$
P_{1}(m, m)=\left(\frac{\lambda_{t h_{2}}}{\ln (2)\left(\lambda_{p_{1}}+\sum_{l=1}^{L} \lambda_{c_{l}}\left|\boldsymbol{\Omega}_{1 r_{l}}(m, m)\right|^{2}\right)}-\frac{\boldsymbol{C}_{\tilde{\boldsymbol{z}}_{2}}(m, m)}{\boldsymbol{S}_{\Psi}^{2}(m, m)}\right)^{+}, \quad P_{2}(m, m)=\left(\frac{\lambda_{t h_{1}}}{\ln (2)\left(\lambda_{p_{2}}+\sum_{l=1}^{L} \lambda_{c_{l}}\left|\boldsymbol{\Omega}_{2 r_{l}}(m, m)\right|^{2}\right)}-\frac{\boldsymbol{C}_{\tilde{z}_{1}}(m, m)}{\boldsymbol{S}_{\Phi}^{2}(m, m)}\right)^{+} .
$$

where $\mathcal{L}$ is the Lagrangian function [27] which is given in (26). $\boldsymbol{\lambda}$ is a vector that contains all the Lagrangian multipliers of the system, where $\lambda_{p_{t}}, \lambda_{c_{l}}, \lambda_{t h_{t}}$ and $\lambda_{B}$, represent the Lagrangian multipliers related to the peak power budget constraint at the $t^{t h} \mathrm{PU}$, peak power budget constraint at the $l^{t h} \mathrm{CU}$, the primary target rate constraint for the $t^{t h} \mathrm{PU}$, and the bandwidth constraint, respectively. It includes also $\lambda_{R_{l}}, l=1, \cdots, L$ related to constraint (23) if the Max-Min utility is used. By taking the derivative of the Lagrangian with respect to the $P_{t}(m, m)$, and $P_{c_{l}}(m, m)$ where $t \in\{1,2\}, m=1, \ldots, M$ and $l=1, \ldots, L$, we can find the optimal primary power allocated to the $m^{t h}$ antenna at $\mathrm{PU}_{t}$ as well as the optimal transmit powers allocated to the $m^{t h}$ antenna at $\mathrm{CU}_{l}$ that maximize the Lagrangian function and, consequently, the cognitive utility rate. Since the primary powers are independent of the cognitive utility expression, we can derive the closed-form expressions of $P_{1}(m, m)$ and $P_{2}(m, m)$ at each iteration for fixed bandwidth and relay amplification factors as given in (27), where $\ln (2)$ is the natural logarithm of 2 . However, the expression of the $l^{\text {th }} \mathrm{CU}$ transmit power depends on the utility approach as follows:

Max C/I Utility: For max C/I utility, the $l^{\text {th }} \mathrm{CU}$ transmit power over the $m^{t h}$ antenna can be expressed as

$$
P_{c_{l}}(m, m)=\left(\frac{1}{\lambda_{c_{l}} \ln (2)}-\frac{\mathcal{N}_{0}}{S_{c_{l}}^{2}(m, m)}\right)^{+} .
$$

We can see from (28) that the value of the $P_{c_{l}}(m, m)$ depends on $\lambda_{c_{l}}$ related to constraint (18) (i.e., corresponding to the primary powers and primary bandwidth). Also, it depends on the channel values between the CUs and cognitive base station. In this approach it is clear that, all resources are allocated to the CUs with favorable channel conditions.

Max-Min Utility: By taking the derivative of the Lagrangian of (22)-(24) with respect to $P_{c_{l}}(m, m)$ and equating it to zero, the $l^{\text {th }} \mathrm{CU}$ transmit power over the $m^{\text {th }}$ antenna can be derived as

$$
P_{c_{l}}(m, m)=\left(\frac{\lambda_{R_{l}}}{\lambda_{c_{l}} \ln (2)}-\frac{\mathcal{N}_{0}}{S_{c_{l}}^{2}(m, m)}\right)^{+}
$$

By taking the derivative of the Lagrangian with respect to $R_{\text {min }}$, we can deduce that $\sum_{l}^{L} \lambda_{R_{l}}=1$, which means that $\lambda_{R_{l}} \in[0,1]$. By comparing (29) with (28), we can see that $\lambda_{R_{l}}$ values control the priority of the resource allocation. However, enhancing the worst channel condition (i.e., corresponding to the minimum rate achieved) could come at the expense of users with good channel conditions which leads to more fairness between the CUs.

Proportional Fair Utility: For PF utility, the $l^{\text {th }}$ CU transmit power over the $m^{\text {th }}$ antenna can be derived as

$$
\begin{aligned}
& P_{c_{l}}(m, m)=\left(\frac{1}{\lambda_{c_{l}} \ln (2)} \prod_{\substack{k=1 \\
k \neq l}}^{L} B_{k} .\right. \\
& \left.\sum_{m=1}^{M} \log _{2}\left(1+\frac{P_{c_{k}}(m, m) \boldsymbol{S}_{c_{k}}^{2}(m, m)}{\mathcal{N}_{0}}\right)-\frac{\mathcal{N}_{0}}{\boldsymbol{S}_{c_{l}}^{2}(m, m)}\right)^{+} .
\end{aligned}
$$

In this approach, a tradeoff between the maximization of the sum rate and the maximization of the minimum rate can be clearly deduced in (30). The $l^{\text {th }} \mathrm{CU}$ transmit power over the $m^{t h}$ antenna $P_{c_{l}}(m, m)$ depends directly on the sum rate of other antennas at the same $\mathrm{CU}$ and the product of the other CUs rates. This approach tries to avoid having any user with very low data rate and maximize the product of the CUs rates simultaneously.

For all utilities, we can employ the subgradient method to find the optimal Lagrangian multipliers of this problem [28]. Hence, to obtain the solution, we can start with any initial values for the different Lagrangian multipliers and evaluate the optimal powers. We then update the Lagrangian multipliers at the next iteration $(i+1)$ as follows

$$
\begin{gathered}
\lambda_{p_{t}}^{(i+1)}=\lambda_{p_{t}}^{(i)}-\delta_{p_{t}}^{(i)}\left(\bar{P}_{p}-\frac{B_{0}}{2} \operatorname{Tr}\left(\boldsymbol{P}_{t}\right)\right), \forall t=1,2, \text { (31) } \\
\lambda_{c_{l}}^{(i+1)}=\lambda_{c_{l}}^{(i)}-\delta_{c_{l}}^{(i)}\left(\bar{P}_{c}-\left(B_{l} \operatorname{Tr}\left(\boldsymbol{P}_{c_{l}}\right)+\right.\right. \\
\left.\left.\frac{B_{0}}{2} \operatorname{Tr}\left(\boldsymbol{\Omega}_{1 r_{l}} \boldsymbol{P}_{1} \boldsymbol{\Omega}_{1 r_{l}}^{H}+\boldsymbol{\Omega}_{2 r_{l}} \boldsymbol{P}_{2} \boldsymbol{\Omega}_{2 r_{l}}^{H}+N_{0} \boldsymbol{G}_{l} \boldsymbol{G}_{l}^{H}\right)\right)\right) \\
\forall l=1, \ldots, L \\
\lambda_{t h_{1}}^{(i+1)}=\lambda_{t h_{1}}^{(i)}-\delta_{t h_{1}}^{(i)}\left(R_{p_{1}}-\alpha R_{0}\right) \\
\lambda_{t h_{2}}^{(i+1)}=\lambda_{t h_{2}}^{(i)}-\delta_{t h_{2}}^{(i)}\left(R_{p_{2}}-(1-\alpha) R_{0}\right) \\
\lambda_{B}^{(i+1)}=\lambda_{B}^{(i)}-\delta_{B}^{(i)}\left(B_{\mathrm{tot}}-\left(\sum_{l=0}^{L} B_{l}\right)\right)
\end{gathered}
$$




$$
\lambda_{R_{l}}^{(i+1)}=\lambda_{R_{l}}^{(i)}-\delta_{R_{l}}^{(i)}\left(R_{c_{l}}-R_{\min }\right), \forall l=1, \ldots, L .
$$

where $\delta_{p_{t}}^{(i)}, \delta_{c_{l}}^{(i)}, \delta_{t h_{1}}^{(i)}, \delta_{t h_{2}}^{(i)}, \delta_{B}^{(i)}$ and $\delta_{R_{l}}^{(i)}$ are the updated step size according to the nonsummable diminishing step length policy (see [28] for more details). The updated values of the optimal powers and the Lagrangian multipliers are repeated until convergence.

\section{B. Particle Swarm Optimization Algorithm}

In the second step, we employ the PSO algorithm to optimize $\boldsymbol{B}$ and $\boldsymbol{G}_{l}, \forall l=1, \ldots, L$. The PSO idea was introduced in 1995 [29] and it is inspired by swarm intelligence, social behavior, and food searching by a flock birds and a school of fish. This approach is widely used in several wireless communication fields due to its simplicity and efficiency [30][32]. Due to the following advantages of PSO compared with the other heuristic approaches, we apply it for solving this problem: (i) simple search process and easy to implement by manipulating few numerical parameters (e.g., such as the number of particles and acceleration factors for PSO) (ii) it requires low computational cost attained from small number of agents; and (iii) it provides a good convergence speed [33]. Thus, we propose to implement it to find near-optimal relay amplification gains and bandwidth fractions.

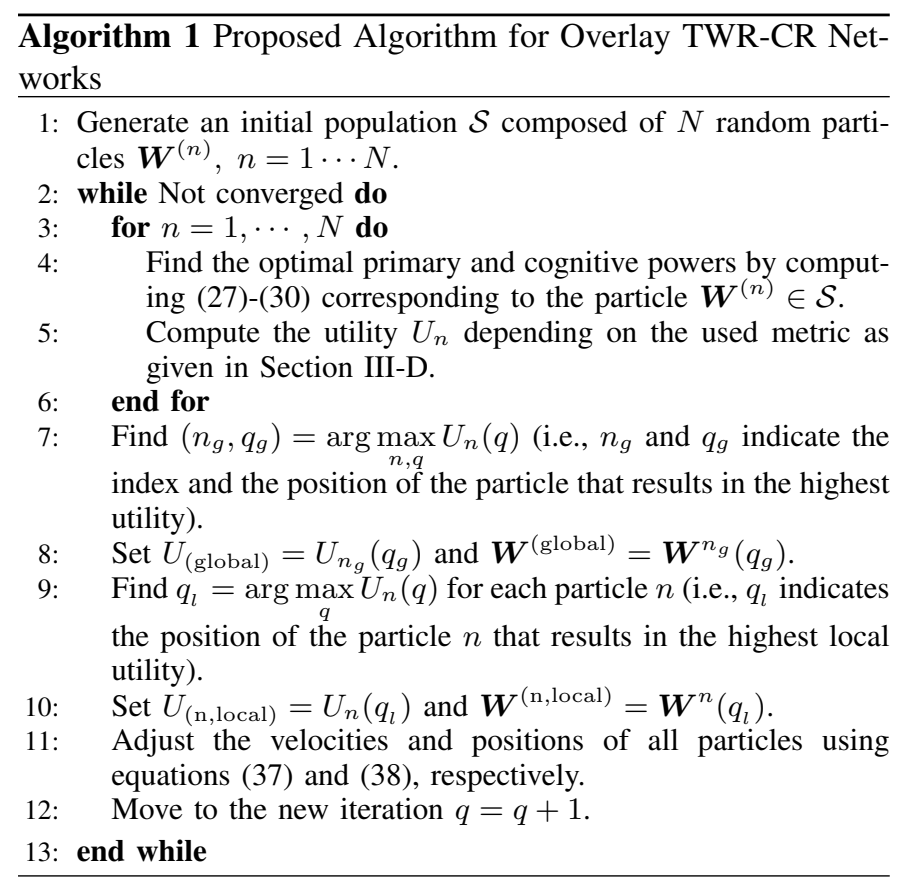

First, the PSO generates $N$ random particles (i.e., a vector contains random $\boldsymbol{B}$ and $\left.\boldsymbol{G}_{l}, \forall l=1, \ldots, L\right) \boldsymbol{W}^{(n)}, \quad n=$ $1, \cdots, N$, of length $1 \times\left(L(2 M)^{2}+(L+1)\right)$ to form an initial population set $\mathcal{S}$, where $(2 M)$ corresponds to the fact that during the PSO algorithm we optimize complex amplification gain matrices of multiple antenna relays. Note that when $M=1$, we focus on optimizing a single real entry per relay: the amplification gain. The algorithm computes the achieved utility (16) of all particles by computing the optimal terminal powers derived in Section IV-A for this $\boldsymbol{W}^{(n)}$. Then, it finds the particle that provides the global optimal utility for this iteration, denoted $\boldsymbol{W}^{\text {(global) }}$. In addition, for each particle $n$, it memorizes the position of its previous best performance, denoted $\boldsymbol{W}^{(\mathrm{n} \text {,local) }}$. After finding these two best values, PSO updates its velocity $\boldsymbol{\nu}_{j}^{(n)}$ and its particle positions $\boldsymbol{W}_{j}^{(n)}$, respectively at each iteration $q$ as follows:

$$
\begin{aligned}
\boldsymbol{\nu}_{j}^{(n)}(q+1)=\psi \boldsymbol{\nu}_{j}^{(n)}(q) & +c_{1} r_{1}\left(\boldsymbol{W}_{j}^{(\mathrm{n}, \text { local })}(q)-\boldsymbol{W}_{j}^{(n)}(q)\right) \\
& +c_{2} r_{2}\left(\boldsymbol{W}_{j}^{(\text {global })}(q)-\boldsymbol{W}_{j}^{(n)}(q)\right),
\end{aligned}
$$

$$
\boldsymbol{W}_{j}^{(n)}(q+1)=\left(\boldsymbol{W}_{j}^{(n)}(q)+\boldsymbol{\nu}_{j}^{(n)}(q+1)\right)^{+},
$$

where $\psi$ is the inertia weight used to control the convergence speed $(0.8 \leq \psi \leq 1.2) . r_{1}$ and $r_{2}$ are two random positive numbers generated for each element $j$. Finally, $c_{1}$ and $c_{2}$ are the step size that a particle takes towards the best individual candidate solution $\boldsymbol{W}^{(\mathrm{n}, \text { local })}$ and the global best solution $W^{\text {(global) }}$. This procedure is repeated until convergence (i.e., the utility remains constant for a certain number of iterations or reaching maximum number of iterations). Details of the proposed algorithm as it is applied to our optimization problem are given in Algorithm 1.

Although PSO's application has been proved to be effective, convergence to its most optimistic solution cannot be guaranteed in theory [34].

\section{Simulation Results}

In this section, we provide selected simulation results for i.i.d Rayleigh fading channels to study the performance of the proposed scheme given in Fig. 1. The total bandwidth and average noise power per unit frequency are assumed to be equal to $B_{\text {tot }}=5 \mathrm{MHz}$ and $\mathcal{N}_{0}=1 \mathrm{Watt} / \mathrm{Hz}$, respectively. The PSO algorithm is executed using these parameters: the initial number of particles is set to $N=30$ and the maximum number of iterations is equal to 200. It is assumed that the PSO algorithm converges when the utility remains constant for 10 consecutive iterations or reaching maximum number of iterations.

\section{A. System Performance}

Fig. 3 plots the achieved cognitive sum rate using PSO algorithm presented in Algorithm 1 versus the rate profile of the system $\alpha$ for different utilities (Max C/I, PF, and MaxMin) with fixed $L=4, R_{0}=10 \mathrm{Mbits} / \mathrm{s}, \bar{P}_{p}=\bar{P}_{c}=20$ $\mathrm{dBm}$ and different number of antennas (i.e., $M=\{1,2\}$ ). It is shown that the optimal $\alpha$ that maximizes the cognitive sum rate is equal to 0.5 (corresponding to the point where the threshold rate at $\mathrm{PU}_{1}$ is equal to the threshold rate at $\mathrm{PU}_{2}$ ). Also, we notice that the sum rate has a symmetric behvior that reflects the symmetry of the TWR. We compare the three utilities with the uniform bandwidth case using maximum sum rate utility (i.e., we choose $B_{0}$ such that it satisfies (10). Then, we consider uniform power distribution among the antennas and distribute the remaining bandwidth to the CUs i.e., $\left.B_{l}=\frac{B_{\text {tot }}-B_{0}}{L}, \forall l=1, \ldots, L\right)$. This figure also shows the improvement of cognitive sum rate thanks to the use of MIMO 
antennas (i.e., $M=2$ ) instead of the single antenna scheme (i.e., $M=1$ ). For instance, for $\alpha=0.5$, we were able to increase the achievable cognitive sum rate by going from 6.10 Mbits/s to around $9.87 \mathrm{Mbits} / \mathrm{s}$, $4.31 \mathrm{Mbits} / \mathrm{s}$ to around 8.05 Mbits/s, and $3.34 \mathrm{Mbits} / \mathrm{s}$ to around $7.06 \mathrm{Mbits} / \mathrm{s}$ by using two antennas instead of a single one for Max C/I, PF, and Max-Min utilities, respectively.

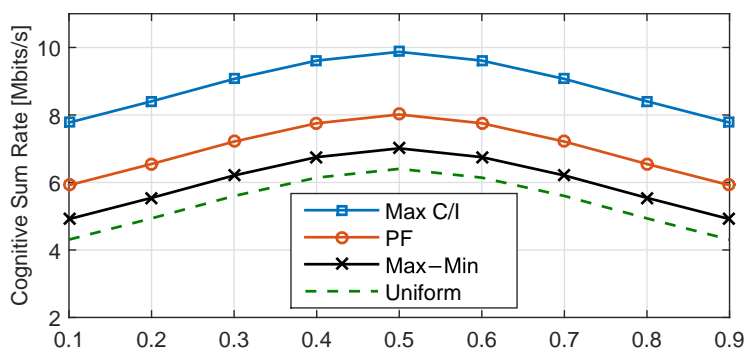

(a)

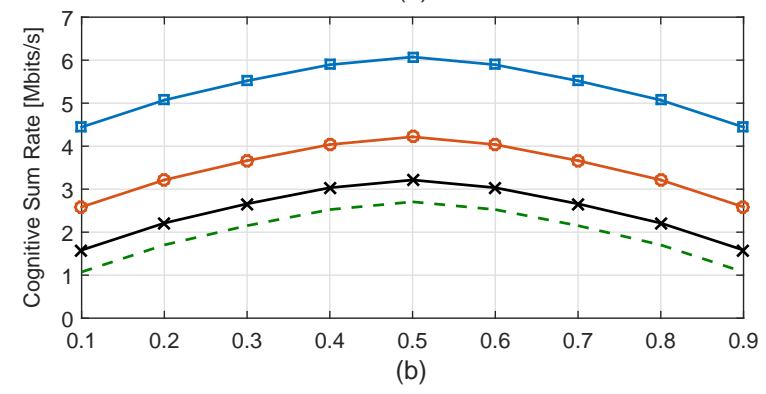

$\alpha$

Fig. 3 Cognitive sum rate as a function of $\alpha$ for different utilities with $L=4, R_{0}=10 \mathrm{Mbits} / \mathrm{s}$, and $\bar{P}_{p}=\bar{P}_{c}=20 \mathrm{dBm}$, for different number of antennas (a) $M=2$, (b) $M=1$.

All simulations show that Max C/I utility leads to the highest sum rate in the network. However, this comes at the expense of fairness as it is shown in Table I. Indeed, the table compares between the different utilities for the same channel realization with fixed $\alpha=0.5, L=4, R_{0}=10 \mathrm{Mbits} / \mathrm{s}$, and $\bar{P}_{p}=\bar{P}_{c}=20 \mathrm{dBm}$. By using one realization, it can be shown that Max C/I enhances the cognitive sum rate, by allocating most of the resources to a unique user having the best channel conditions (i.e., $\mathrm{CU}_{3}$ for $M=1$ and $\mathrm{CU}_{2}$ for $M=2$ ). On the other hand, the PF approach maximizes the geometric mean for all the users by allocating almost the same amount of bandwidths to CUs, while Max-Min approach maximizes the minimum cognitive rate and provides the same rate for all cognitive users, hence, leads to fairness performance. The choice of the utility is related to the service used by the CUs. For instance, if the application requires same uplink rates MaxMin utility can be used. However, if it consists in a pure cognitive transmission without priorities, then Max C/I could be employed.

In Fig. 4, we aim to investigate the impact of the power budget constraint on the system performance. In this figure, we plot the cognitive sum rate for all the utilities versus the power budget $\left(\bar{P}_{p}=\bar{P}_{c}\right)$ with $\alpha=0.5, L=4, M=2$, and different values of $R_{0}=\{10,20\}$ Mbits/s. It is shown that increasing the target rate $R_{0}$ for the same power budget reduces the cognitive sum rate, since satisfying constraints (19) and (20) requires more bandwidth (i.e., higher $B_{0}$ ) as Fig. 4(b)
TABLE I: Strategy of cognitive users corresponding to each utility with $\alpha=0.5, L=4, R_{0}=10 \mathrm{Mbits} / \mathrm{s}$, and $\bar{P}_{p}=\bar{P}_{c}=20 \mathrm{dBm}$.

\begin{tabular}{|c||c|c|c|c|c|c|}
\hline \multicolumn{1}{|c||}{} & \multicolumn{3}{c|}{ M=1 } & \multicolumn{3}{c|}{ M=2 } \\
\cline { 2 - 7 } & Max C/I & PF & Max-Min & Max C/I & PF & Max-Min \\
\hline$\sum_{l=1}^{L} R_{c_{l}}[\mathrm{Mbits} / \mathrm{s}]$ & 6.51 & 4.83 & 3.64 & 9.87 & 8.05 & 7.06 \\
\hline$R_{c_{1}}[\mathrm{Mbits} / \mathrm{s}]$ & $\sim 0$ & 0.87 & 0.91 & $\sim 0$ & 2.01 & 1.77 \\
\hline$R_{c_{2}}[\mathrm{Mbits} / \mathrm{s}]$ & $\sim 0$ & 1.39 & 0.91 & 9.87 & 2.35 & 1.77 \\
\hline$R_{c_{3}}[\mathrm{Mbits} / \mathrm{s}]$ & 6.51 & 1.37 & 0.91 & $\sim 0$ & 1.95 & 1.77 \\
\hline$R_{c_{4}}[\mathrm{Mbits} / \mathrm{s}]$ & $\sim 0$ & 1.19 & 0.91 & $\sim 0$ & 1.74 & 1.77 \\
\hline$B_{0}[\%]$ & 41.90 & 44.96 & 44.91 & 31.85 & 33.24 & 33.44 \\
\hline$B_{1}[\%]$ & $\sim 0$ & 13.76 & 17.16 & $\sim 0$ & 16.69 & 19.56 \\
\hline$B_{2}[\%]$ & $\sim 0$ & 13.76 & 12.51 & 67.59 & 16.69 & 12.78 \\
\hline$B_{3}[\%]$ & 58.10 & 13.76 & 10.61 & $\sim 0$ & 16.69 & 19.01 \\
\hline$B_{4}[\%]$ & $\sim 0$ & 13.76 & 14.81 & $\sim 0$ & 16.69 & 15.21 \\
\hline
\end{tabular}

shows, as well as higher $\boldsymbol{P}_{1}$ and $\boldsymbol{P}_{2}$ values. As a result, CUs are forced to reduce their transmitted power $\boldsymbol{P}_{c_{l}}, \forall 1, \ldots, L$, to support primary transmission and respect constraint (18). For instance, for $\bar{P}=10 \mathrm{dBm}$ with Max $\mathrm{C} / \mathrm{I}$ approach, the total cognitive sum rate is reduced by around $20 \%$ by going from around $6.00 \mathrm{Mbits} / \mathrm{s}$ to around $4.80 \mathrm{Mbits} / \mathrm{s}$ using $R_{0}=20$ Mbits/s instead of $R_{0}=10 \mathrm{Mbits} / \mathrm{s}$. On the other hand, we can see that increasing the power budget will provide more bandwidth to secondary users to accomplish their transmission. For instance, with $10 \mathrm{dBm}$, primary transmission needs $60 \%$ of the total bandwidth whereas with $30 \mathrm{dBm}$ only $20 \%$ of the bandwidth is needed using Max C/I utility. Thus, the secondary sum rate is multiplied by 2 .
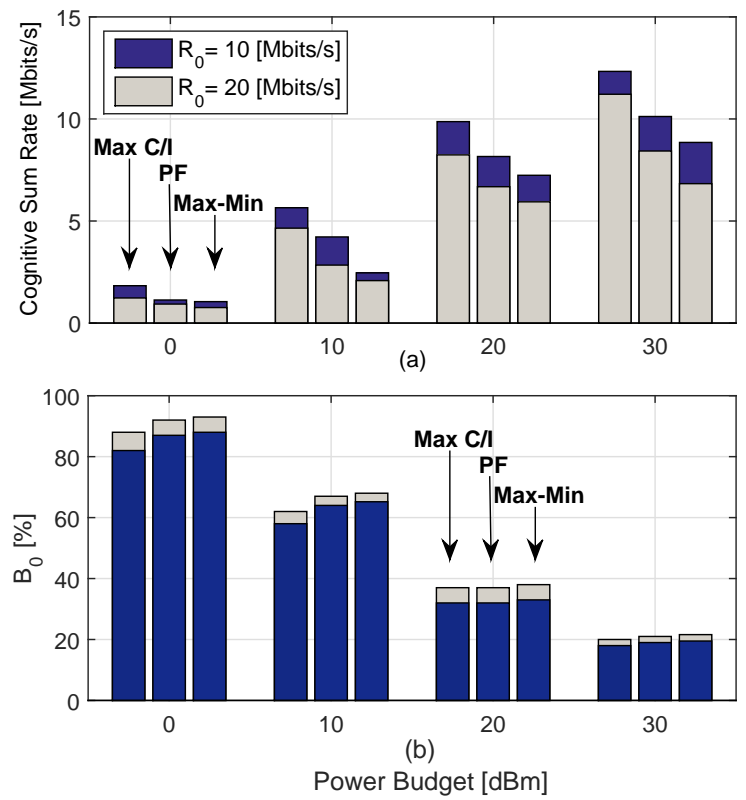

Fig. 4 (a) Cognitive sum rate, (b) primary bandwidth fraction $\left(B_{0}\right.$ [\%]) versus power budget constraint $\left(\bar{P}_{p}=\bar{P}_{c}\right)$, for $\alpha=0.5, L=$ $4, M=2$.

Fig. 5 illustrates the cognitive sum rate as a function of $M$ for $\alpha=0.5, L=4, R_{0}=10 \mathrm{Mbits} / \mathrm{s}$, and $\bar{P}_{p}=\bar{P}_{c}=20$ $\mathrm{dBm}$. From this figure, we can deduce that the achievable rate is improving when $M$ increases, in other words, MIMO antennas provide more degrees of freedom to the system which enhances the cognitive sum rate. Similar to the power budget effect, increasing the number of antennas offers more 

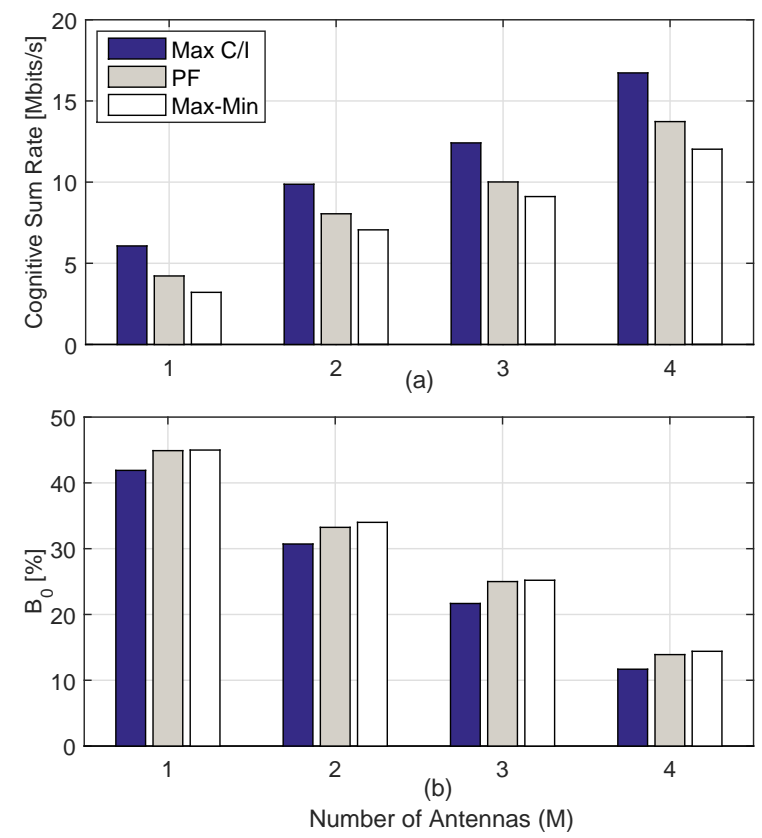

Fig. 5 (a) Cognitive sum rate, (b) primary bandwidth fraction $\left(B_{0}\right.$ [\%]), versus number of antennas for $\alpha=0.5, L=4, R_{0}=10$ Mbits/s, and $\bar{P}_{p}=\bar{P}_{c}=20 \mathrm{dBm}$.

bandwidth to secondary transmissions. Indeed, with MIMO antennas, primary transmission is enhanced as it demands less relay power and thus more power can be allocated to secondary transmissions.

\section{B. Convergence Speed}

In Fig. 6, we compare between the performance of PSO and a recently proposed meta-heuristic approach entitled GWO, which are both employed with the primal-dual method, by investigating their convergence speed defined by the number of iterations needed to reach convergence. Note that an iteration in Fig. 6 corresponds to one iteration of the "while loop" given in Algorithm 1 line 2-13. GWO is inspired by grey wolf hunt. It mimics the leadership hierarchy and hunting mechanism of grey wolves in nature. During an iteration, the algorithm categorizes the candidates (i.e., grey wolves) into four groups for simulating the leadership hierarchy: Group 1 corresponds to the fittest solution, Group 2 and 3 are the second and third best solutions. Finally, Group 4 contains the remaining candidates of the population. Also, the algorithm simulates the hunting, searching for prey, encircling prey, and attacking prey of grey wolves. For example, the hunting corresponds to the position update of each candidate from an iteration to another (see [22] for more details). We plot the cognitive sum rate versus the number of iterations for PSO and GWO. It can be clearly seen that PSO achieves its suboptimal solution faster than GWO. For instance, it requires around 10-20 iterations to converge with the Max C/I utility while GWO needs 30-40 iterations. Moreover, we can notice that PSO reaches a better suboptimal solution than GWO.

For a given maximum number of iterations of the subgradient method $I_{\max }$, the complexity is given by $\min \left(I_{\max }, 1 / \epsilon^{2}\right)$,
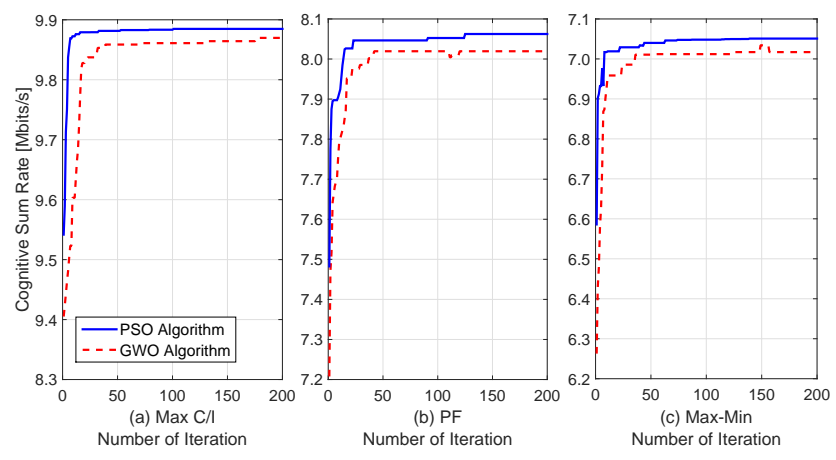

Fig. 6 Algorithms convergence speed for $\alpha=0.5, L=4, M=$ $2, R_{0}=10 \mathrm{Mbits} / \mathrm{s}$, and $\bar{P}_{p}=\bar{P}_{c}=20 \mathrm{dBm}$.

where $\epsilon$ represents the accuracy-guarantee which is defined by the difference between the best value and the iterate value [27]. According to (31)-(36), subgradient needs to calculate $12 M L+8 M+16 L+15$ multiplications and $6 M L+8 M+9 L+12$ additions at each iteration. These operations are computed for each particle of the meta-heuristic approach. On the other hand, PSO and GWO are two metaheuristic algorithms where the exact number of iterations needed to reach the solution is arbitrary and depends on the studied scenario. However, the computational complexity per iteration can be determined. According to (37) and (38), PSO needs to calculate 5 multiplications and 5 additions for every element of $\boldsymbol{W}^{(n)}$. Hence, $5\left(L(2 M)^{2}+(L+1)\right) N$ multiplications and $5\left(L(2 M)^{2}+(L+1)\right) N$ additions are calculated every iteration for the total $N$ particles whereas GWO calculates $13\left(L(2 M)^{2}+(L+1)\right) N$ multiplications and $11\left(L(2 M)^{2}+(L+1)\right) N$ additions according to [22]. In our simulation results, we set $\epsilon=0.1$ and $I_{\max }=200$ iterations for subgradient algorithm. While PSO and GWO algorithms are executed for at most 200 iterations (i.e., the utilities are computed at most $200 \times N$ times), they are stopped if the achieved utility remains constant for a certain number of consecutive iterations.

TABLE II CPU times (sec) and number of iterations for the proposed joint-optimization method. The first row shows the total CPU times, while the second row shows the number of iterations needed when solving the joint power, bandwidth, and amplification factors problem.

\begin{tabular}{|c||c|c|c|c|c|c|}
\hline \multicolumn{1}{|c||}{} & \multicolumn{2}{c|}{ Max C/I } & \multicolumn{2}{c|}{ PF } & \multicolumn{2}{c|}{ Max-Min } \\
\cline { 2 - 7 } & PSO & GWO & PSO & GWO & PSO & GWO \\
\hline Total CPU time & 39 & 59 & 57 & 86 & 76 & 121 \\
\hline$I^{*}$ & 15 & 32 & 22 & 48 & 16 & 39 \\
\hline
\end{tabular}

For 200 realizations, $N=30, L=4$ and $M=2$, results show that on average PSO is faster than GWO and requires less time to converge as shown in Table II. In Table II, we compute the CPU times in seconds of both algorithms and record the iteration number (denoted by $I^{*}$ ) needed to reach the near optimal solution of the joint optimization (i.e., optimizing the power, bandwidth, and relay amplification matrices), which exactly marks the instant when the algorithm achieves its steady state utility. Increasing the number of particles $N$ would enhance the convergence speed of the algorithms. In fact, PSO 
and GWO are able to achieve their solutions with a lower number of iterations but they require more CPU times as they need to perform more additions and multiplications during each iteration. Note that all tests were performed on a laptop machine featuring an Intel(R) Core(TM) i7 CPU and running Windows 8.1. The clock of the machine is set to $2.66 \mathrm{GHz}$ with a 8 GB memory.

\section{CONCLUSION}

In this paper, we investigated multiple-input multiple-output two-way relaying scheme for overlay cognitive radio networks where cognitive users are engaged to support primary transmission over a fraction of the bandwidth. More specifically, we considered multiple amplify-and-forward relays where the primary and cognitive terminal powers were optimized adaptively with the bandwidth and amplification gains. The objective was based on maximizing the cognitive utility while satisfying a certain primary target rate. Starting with expressions of primary and cognitive powers for fixed bandwidths and amplification gains, we employed the heuristic particle swarm optimization algorithm to reach a near-optimal solution. Moreover, in addition to the sum rate objective function, we investigated other utilities that introduce more fairness among cognitive users. In our ongoing task, we are trying to develop an approximation algorithm to solve the formulated optimization problem.

\section{REFERENCES}

[1] S. Haykin, "Cognitive radio: Brain-empowered wireless communications," IEEE Journal on Selected Areas in Communications, vol. 23, no. 2, pp. 201-220, Feb. 2005.

[2] A. Goldsmith, S. Jafar, I. Maric, and S. Srinivasa, "Breaking spectrum gridlock with cognitive radios: An information theoretic perspective," Proceedings of the IEEE, vol. 97, no. 5, pp. 894-914, May 2009.

[3] J. Mitola III, Cognitive Radio: An Integrated Agent Architecture for Software Defined Radio. Ph.D. Dissertation, Royal Institute of Technology (KTH), Stockholm, Sweden, 2000.

[4] A. Alsharoa, F. Bader, and M. Alouini, "Relay selection and resource allocation for two-way df-af cognitive radio networks," IEEE Wireless Communications Letters, vol. 2, no. 4, pp. 427-430, Aug. 2013.

[5] W. Su, J. Matyjas, and S. Batalama, "Active cooperation between primary users and cognitive radio users in heterogeneous Ad-Hoc networks," IEEE Transactions on Signal Processing, vol. 60, no. 4, pp. 1796-1805, Apr. 2012.

[6] B. Rankov and A. Wittneben, "Spectral efficient protocols for halfduplex fading relay channels," IEEE Journal on Selected Areas in Communications, vol. 25, no. 2, pp. 379-389, Feb. 2007.

[7] K. Jitvanichphaibool, R. Zhang, and Y.-C. Liang, "Optimal resource allocation for two-way relay-assisted OFDMA," IEEE Transactions on Vehicular Technology, vol. 58, no. 7, pp. 3311-3321, Sep. 2009.

[8] K.-J. Lee, H. Sung, E. Park, and I. Lee, "Joint optimization for one and two-way MIMO AF multiple-relay systems," IEEE Transactions on Wireless Communications, vol. 9, no. 12, pp. 3671-3681, Dec. 2010.

[9] Y. Rong, "Joint source and relay optimization for two-way MIMO multi-relay networks," IEEE Communications Letters, vol. 15, no. 12, pp. 1329-1331, Dec. 2011.

[10] A. Alsharoa, H. Ghazzai, and M.-S. Alouini, "Near optimal power allocation with PSO algorithm for MIMO cognitive networks using multiple AF two-way relays," in Proc. of the IEEE International Conference on Communications (ICC'2014), Sydney, Australia, Jun. 2014.

[11] A. Alsharoa, H. Ghazzai, and M.-S. Alouini, "Efficient multiple antennarelay selection algorithms for MIMO unidirectional-bidirectional cognitive relay networks," Transactions on Emerging Telecommunications Technologies (ETT), Apr. 2014

[12] S. Toroujeni, S. Sadough, and S. Ghorashi, "Spectrum leasing for OFDM-Based cognitive radio networks," IEEE Transactions on Vehicular Technology, vol. 62, no. 5, pp. 2131-2139, Jun. 2013.
[13] S. Jayaweera and T. Li, "Dynamic spectrum leasing in cognitive radio networks via primary-secondary user power control games," IEEE Transactions on Wireless Communications, vol. 8, no. 6, pp. 3300-3310, Jun. 2009.

[14] F. Gomez-Cuba, R. Asorey-Cacheda, F. Gonzalez-Castano, and H. Huang, "Application of cooperative diversity to cognitive radio leasing: Model and analytical characterization of resource gains," IEEE Transactions on Wireless Communications, vol. 12, no. 1, pp. 40-49, Jan. 2013

[15] O. Simeone, I. Stanojev, S. Savazzi, Y. Bar-Ness, U. Spagnolini, and R. Pickholtz, "Spectrum leasing to cooperating secondary Ad Hoc networks," IEEE Journal on Selected Areas in Communications, vol. 26, no. 1, pp. 203-213, Jan. 2008

[16] E. Biglieri, A. Goldsmith, L. Greenstein, N. Mandayam, and H. Poor, Principles of cognitive radio. New York, NY, USA: Cambridge University Press, 2012.

[17] Y. Pei and Y.-C. Liang, "Resource allocation for device-to-device communications overlaying two-way cellular networks," IEEE Transactions on Wireless Communications, vol. 12, no. 7, pp. 3611-3621, Jul. 2013.

[18] Q. Li, S. H. Ting, A. Pandharipande, and Y. Han, "Cognitive spectrum sharing with two-way relaying systems," IEEE Transactions on Vehicular Technology, vol. 60, no. 3, pp. 1233-1240, Mar. 2011.

[19] A. Hyadi, E. Driouch, W. Ajib, and M.-S. Alouini, "Overlay cognitive radio systems with adaptive two-way relaying," in Proc. of the IEEE Global Communications Conference (GLOBECOM'2013), Atlanta, Georgia, USA, Nov. 2013.

[20] A. Alizadeh, S.-S. Sadough, and N. Khajavi, "Optimal beamforming in cognitive two-way relay networks," in Proc. of the IEEE 21st International Symposium on Personal Indoor and Mobile Radio Communications (PIMRC'2010), Istanbul, Turkey, Sep. 2010.

[21] A. Alsharoa, H. Ghazzai, E. Yaacoub, and M.-S. Alouini, "Bandwidth and power allocation for two-way relaying in overlay cognitive radio systems," in Proc. of the IEEE Global Communications Conference (GLOBECOM'2014), Austin, Texas, USA, Dec. 2014.

[22] S. Mirjalili, S-M. Mirjalili, and A. Lewis, "Grey wolf optimizer," Elsevier Advances in Engineering Software, vol. 69, no. 1, pp. 46-61, Mar. 2014.

[23] S. Chang and B. Kelley, "An efficient time synchronization scheme for broadband two-way relaying networks based on physical-layer network coding," IEEE Communications Letters, vol. 16, no. 9, pp. 1416-1419, Sep. 2012.

[24] X. Bi, J. Zhang, Y. Wang, and P. Viswanath, "Fairness improvement of maximum C/I scheduler by dumb antennas in slow fading channel," in Proc. of the 72nd IEEE Vehicular Technology Conference (VTC Fall'2010), Ottawa, Ontario, Canada, Sep. 2010.

[25] Y. Song and G. Li, "Cross-layer optimization for OFDM wireless networks-Part I: Theoretical framework," IEEE Transactions on Wireless Communications, vol. 4, no. 2, pp. 614-624, Apr. 2005.

[26] T. Zhang, Z. Zeng, and Y. Qiu, "A subcarrier allocation algorithm for utility proportional fairness in OFDM systems," in Proc. of the 67th IEEE Vehicular Technology Conference (VTC Spring'2008), Singapore City, Singapore, pp. 1901-1905, May 2008.

[27] S. Boyd and L. Vandenberghe, Convex Optimization. New York, NY, USA: Cambridge University Press, 2004.

[28] S. Boyd and A. Mutapcic, "Stochastic Subgradient Methods." Notes for EE364, Stanford University, Winter 2006-07.

[29] J. Kennedy and R. Eberhart, "Particle swarm optimization," in Proc. of the IEEE International Conference on Neural Networks, Perth, Australia, Nov/Dec. 1995.

[30] H. Chen, C. Tse, and J. Feng, "Minimizing effective energy consumption in multi-cluster sensor networks for source extraction," IEEE Transactions on Wireless Communications, vol. 8, no. 3, pp. 1480-1489, Mar. 2009.

[31] J. Zhang, S. Chen, X. Mu, and L. Hanzo, "Evolutionary-algorithmassisted joint channel estimation and turbo multiuser detection/decoding for OFDM/SDMA," IEEE Transactions on Vehicular Technology, vol. 63, no. 3, pp. 1204-1222, Mar. 2014.

[32] S. Efazati and P. Azmi, "Effective capacity maximization in multirelay networks with a novel cross-layer transmission framework and powerallocation scheme," IEEE Transactions on Vehicular Technology, vol. 63, no. 4, pp. 1691-1702, May 2014.

[33] M. Clerc and J. Kennedy, "The particle swarm-explosion, stability, and convergence in a multidimensional complex space," IEEE Transactions on Evolutionary Computation, vol. 6, no. 3, pp. 58-73, Feb. 2002.

[34] Q. Bai, "Analysis of particle swarm optimization algorithm," Computer and Information Science, vol. 3, no. 1, Feb. 2010. 


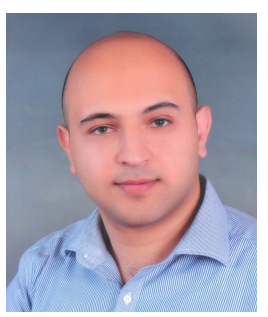

Ahmad Alsharoa (S'12) was born in Irbid, Jordan. He received the B.Sc degree (with honors) from Jordan University of Science and Technology (JUST), Irbid, Jordan, in 2011 and the M.Sc. degree from King Abdullah University of Science and Technology (KAUST) in 2013 both in Electrical Engineering. He is currently pursuing the Ph.D. degree in the Electrical and Computer Engineering with Iowa State University (ISU), Ames, Iowa, US. His current research interests include: energy harvesting, cognitive radio networks, cooperative relay networks, MIMO communications, energy efficient power allocation, and green wireless sensor networks.

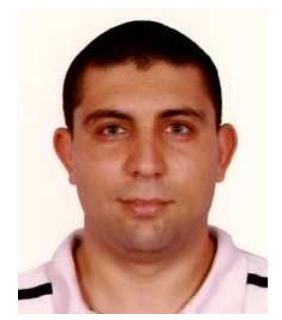

Hakim Ghazzai (S'12, M'15) was born in Tunisia. $\mathrm{He}$ is currently working as a research scientist at Qatar Mobility Innovations Center (QMIC), Doha, Qatar. He received his $\mathrm{PhD}$ degree in Electrical Engineering from King Abdullah University of Science and Technology (KAUST) in Saudi Arabia. $\mathrm{He}$ received his Diplome d'Ingenieur in telecommunication engineering with highest distinction from the Ecole Superieure des Communications de Tunis (SUP'COM), Tunis, Tunisia in 2010 and his Master degree in High-Rate Transmission Systems from the same institute in 2011. His general research interests are at the intersection of wireless networks, green communications and optimization.

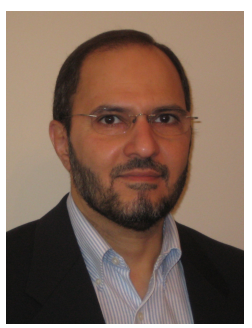

Ahmed E. Kamal (S'82, M'87, SM'91, F'12) is a professor of Electrical and Computer Engineering at Iowa State University in the USA. He received a B.Sc. (distinction with honors) and an M.Sc. both from Cairo University, Egypt, and an M.A.Sc. and a $\mathrm{Ph} . \mathrm{D}$. both from the University of Toronto, Canada, all in Electrical Engineering. He is a Fellow of the IEEE and a senior member of the Association of Computing Machinery. He was an IEEE Communications Society Distinguished Lecturer for 2013 and 2014.

Kamal's research interests include cognitive radio networks, optical networks, wireless sensor networks, and performance evaluation. He received the 1993 IEE Hartree Premium for papers published in Computers and Control in IEE Proceedings, and the best paper award of the IEEE Globecom 2008 Symposium on Ad Hoc and Sensors Networks Symposium.

Kamal chaired or co-chaired Technical Program Committees of several IEEE sponsored conferences including the Optical Networks and Systems Symposia of the IEEE Globecom 2007 and 2010, the Cognitive Radio and Networks Symposia of the IEEE Globecom 2012 and 2014, and the Access Systems and Networks track of the IEEE International Conference on Communications 2016. He is on the editorial boards of the IEEE Communications Surveys and Tutorials, the Computer Networks journal, and the Optical Switching and Networking journal.

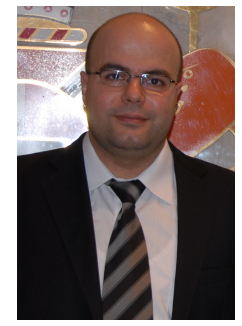

Elias Yaacoub (S'07, M'10, SM'14) he received the B.E. degree in Electrical Engineering from the Lebanese University in 2002, the M.E. degree in Computer and Communications Engineering from the American University of Beirut (AUB) in 2005, and the $\mathrm{PhD}$ degree in Electrical and Computer Engineering from AUB in 2010. He served as a Research Assistant in the American University of Beirut from 2004 to 2005, and in the Munich University of Technology in Spring 2005. From 2005 to 2007 , he worked as a Telecommunications Engineer with Dar Al-Handasah, Shair and Partners. From November 2010 till December 2014, he worked as a Research Scientist at the Qatar Mobility Innovations Center (QMIC). Afterwards, he joined Strategic Decisions Group (SDG) where he is working as a Consultant. He is also an Adjunct Research Professor at the Arab Open University (AOU). His research interests include Wireless Communications, Resource Allocation in Wireless Networks, Intercell Interference Mitigation Techniques, Antenna Theory, Sensor Networks, and Bioinformatics.

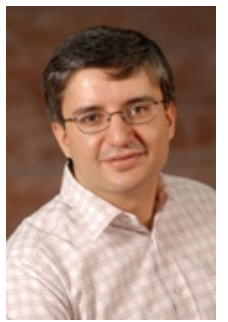

Mohamed-Slim Alouini (S'94, M'98, SM'03, F'09) was born in Tunis, Tunisia. He received the Ph.D. degree in Electrical Engineering from the California Institute of Technology (Caltech), Pasadena, CA, USA, in 1998. He served as a faculty member in the University of Minnesota, Minneapolis, MN, USA, then in the Texas A\&M University at Qatar, Education City, Doha, Qatar before joining King Abdullah University of Science and Technology (KAUST), Thuwal, Makkah Province, Saudi Arabia as a Professor of Electrical Engineering in 2009. His current research interests include the modeling, design, and performance analysis of wireless communication systems. 